\title{
Half-face based recognition using principal component analysis
}

\author{
Ahmed M. Alkababji ${ }^{1}$, Sara Raed Abd ${ }^{2}$ \\ ${ }^{1}$ Department of Computer Engineering, University of Mosul, Iraq \\ ${ }^{2}$ Technical Computer Engineering Department, Alhadbaa University College, Mosul, Iraq
}

\begin{tabular}{l}
\hline \hline Article Info \\
\hline Article history: \\
Received Oct 8, 2020 \\
Revised May 26, 2021 \\
Accepted Jun 1, 2021 \\
\hline
\end{tabular}

\section{Keywords:}

Euclidan distance

Face recognition

Half face

PCA

Real time

\begin{abstract}
Face recognition is a considerable problem in the field of image processing. It is used daily in various applications from personal cameras to forensic investigations. Most of the provides solutions proposed based on full-face images, are slow to compute and need more storage. In this paper, we propose an effective way to reduce the features and size of the database in the face recognition method and thus we get an increase in the speed of discrimination by using half of the face. Taking advantage of face symmetry, the first step is to divide the face image into two halves, then the left half is processed using the principal component analysis (PCA) algorithm, and the results are compared by using Euclidian distance to distinguish the person. The system was trained and tested on ORL database. It was found that the accuracy of the system reached up to $96 \%$, and the database was minimized by $46 \%$ and the running time was decreased from $120 \mathrm{msec}$ to $70 \mathrm{msec}$ with a $41.6 \%$ reduction.
\end{abstract}

This is an open access article under the CC BY-SA license.

\section{Corresponding Author:}

Ahmed M. Alkababjir

Department of Computer Engineering

University of Mosul

Mosul, Iraq

Email: ahmedalkababji72@uomosul.edu.iq

\section{INTRODUCTION}

One of the eminent applications in analyzing images in pattern recognition and computer vision is face recognition distinguishes itself to be. Its work is based on the variation of facial characteristics. Face recognition can be found in various fields in our life starting from high security applications to opening our phone. Two key important technologies to solve the problems of face recognition are the extraction of the characteristics of face images and the selection of a suitable classifier are. Facial features can differentiate persons depending on their distinct characteristics. Several factors can affect the appearance of an individual, for example lighting conditions, brightness variations, different emotions, and aging factors during image acquisition, all of these aspects affect the appearance of a person and make it hard to be recognized [1]-[5].

Principal component analysis (PCA) is commonly used for the processing of facial images, which takes advantage of characteristic features called "Eigenfaces". Using Eigenfaces it tries to create a "principal component" image from the training set by aligning the mouth, eyes and other facial features of the subjects in the scanned images, keeping in mide that the gallery and probe images must be normalized in the same size. Then, a method is used in reducing the dimensionality of data by image compression means and providing a structure of the most effective low dimension of facial pattern.

After this reduction any irrelevant information is dropped and the facial structure is decomposed into uncorrelated (orthogonal) components these components are identified as Eigenfaces. A weighted sum feature vector of eigenfaces characterize each face image. These eigenfaces are kept in a one-dimensional array, which for a set of gallery images will result in a two-dimensional array. The image under investigation is compared with the gallery image. The matching result is declared after a computation of the distance between the image 
feature vector and the gallery. This linear method is widely used in face recognition, which by lower dimension representation aims in solving the problem of recognition [6], [7].

Many researchers have contributed various ideas and methods to distinguish people by face. This is because face recognition is an easy way to distinguish people in a short time and with good accuracy. Halvi et al. [8], the authors suggested a way to distinguish faces through one-dimensional (1D) transform domain, which is optioned by transforming the two-dimensional image face into 1D. The features extraction from discrete wavelet transform (DWT) and fast Fourier transform (FFT) are compared with dataset using Euclidian distance (ED) to distinguish faces. Various gradient and Laplacian model for face recognition is proposed by the authors in [9], feature extraction is achieved using linear regression. An edge detection filter converts the face images to binary as preprocessing then each image is divided into segments. Next PCA is used for feature extraction. Finally, a trained artificial neural network on these features is the tool for classification purposes. Chu et al. [10] the author benefits the fact of facial symmetry by proposing a multiple feature subspace analysis (MFSA) approach. The first division of the face image is about the bilateral symmetry axis then several local face patches are obtained by further partitioning. Patches are then labeled using a k-NN classifier in each subspace. Kute et al. [11] the authors propose a novel approach, they claimed that parts of the face (ear, lips and nose) have common information with the whole face in spite the fact that they are form different domains. Hua et al. [12], presented a robust face recognition method where for extracting the vectors of a feature with pose invariance and scale invariance from face images the speeded-up robust feature (SURF) algorithm is used. PCA is then used in producing a new feature space as PCA-SURF local from the SURF feature vectors. Finally, the feature points are cluster by the K-means algorithm, the face images are classified by combing the global and local similarity. Gumus et al. [13], the authors used an Eigenfaces method which is PCA-based and wavelet decomposition to extract features. After generating feature vectors, support vector machine (SVM) and distance classifiers are used for the classification stage.

Face recognition using half the face is not a new technique, over the last two decades starting at 2003 a patent published by [14], [15] used left and right half images of the face overcome the problems caused by directional or non-uniform illumination. In the same year [16] solved the problem of detection of faces with large depth rotations by using half-face templates. More recently, [17] increased the accuracy of threedimensional face recognition depending on face symmetry. Later, [18] showed in their experiment that a solution to the problem of large angle in side face images is using the half-face template. Elastic bunch Graph matching algorithm was used by [19] for half-face recognition. Shehzad et al. [20] a good half-face based recognition system was proposed, but the computation time was too long and the reduction in database size was not studied. Last year, [21] published those researchers from the University of Bradford claim that in face recognition technology a half-face is enough. After studying the literature, it was concluded that the need of a simple, real time (fast) face recognition system is still a scope of research. Moreover, the advantage of data size reduction which effect storage requirements as well as the speed of data transfer on networks, by sending half-face data from camera.

\section{THE PROPOSED SYSTEM}

Motivated by the advantages of reducing the database size, which effect the speed of computation, as well as, minimizing storage area in face recognition, the idea of taking half the face is explored. Can we imagine that if we store face images of a complete city or country, we would need a memory of a very large size to store this database. However, through the proposed system, we can reduce the size of the database to half by storing a half-face for each person's image because a person has two nearly identical halves when his face is divided vertically into two halves. The results proved through the Matlab program that we can distinguish people through only half of the face. The proposed system can be explained in three stages.

\subsection{Pre-processing}

In the first stage, suppose the image face I (x,y) be a two-dimensional intensity value array of size A*N. The original image was of $95 \times 112$ pixels size. Which is then cropped to a $45 \times 112$ pixels image in database. Then the image is converted to a vector of dimension 5,040. Figure 1 shows this conversion. The source image (I), shown in Figure 2 is cut to the size of MxN through the function of Matlab imcrop (image [47 092 112]); which cut the image face to half-face. The obtained result $\left(I^{\prime}\right)$ shown in Figure 2.

The result image $I^{\prime}$ of crop is entered to PCA, feature extraction as well as dimensionality reduction is achieved by PCA. The similarity between the image under test and the images in the gallery (one by one) is the value of the calculated Euclidean distance between their vectors in the feature space. The minimum the value of Euclidean distance the closest is the two images to each other. Figure 3 shows the flowchart of the propose system. 


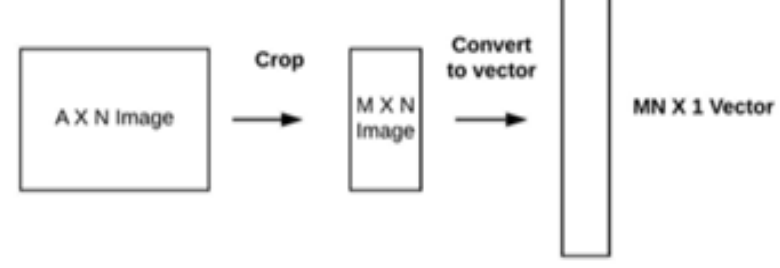

Figure 1. Conversion of image from $\mathrm{A} \times \mathrm{N}$ to $\mathrm{MN} \times 1$ vector

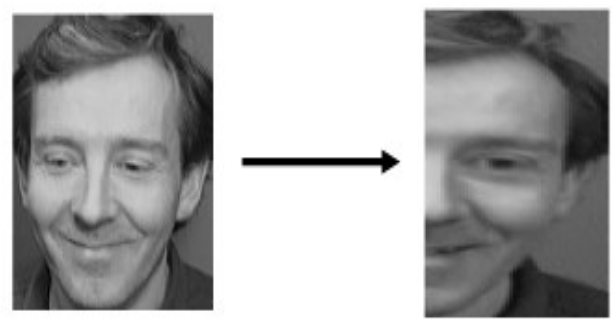

Figure 2. Crop the input image

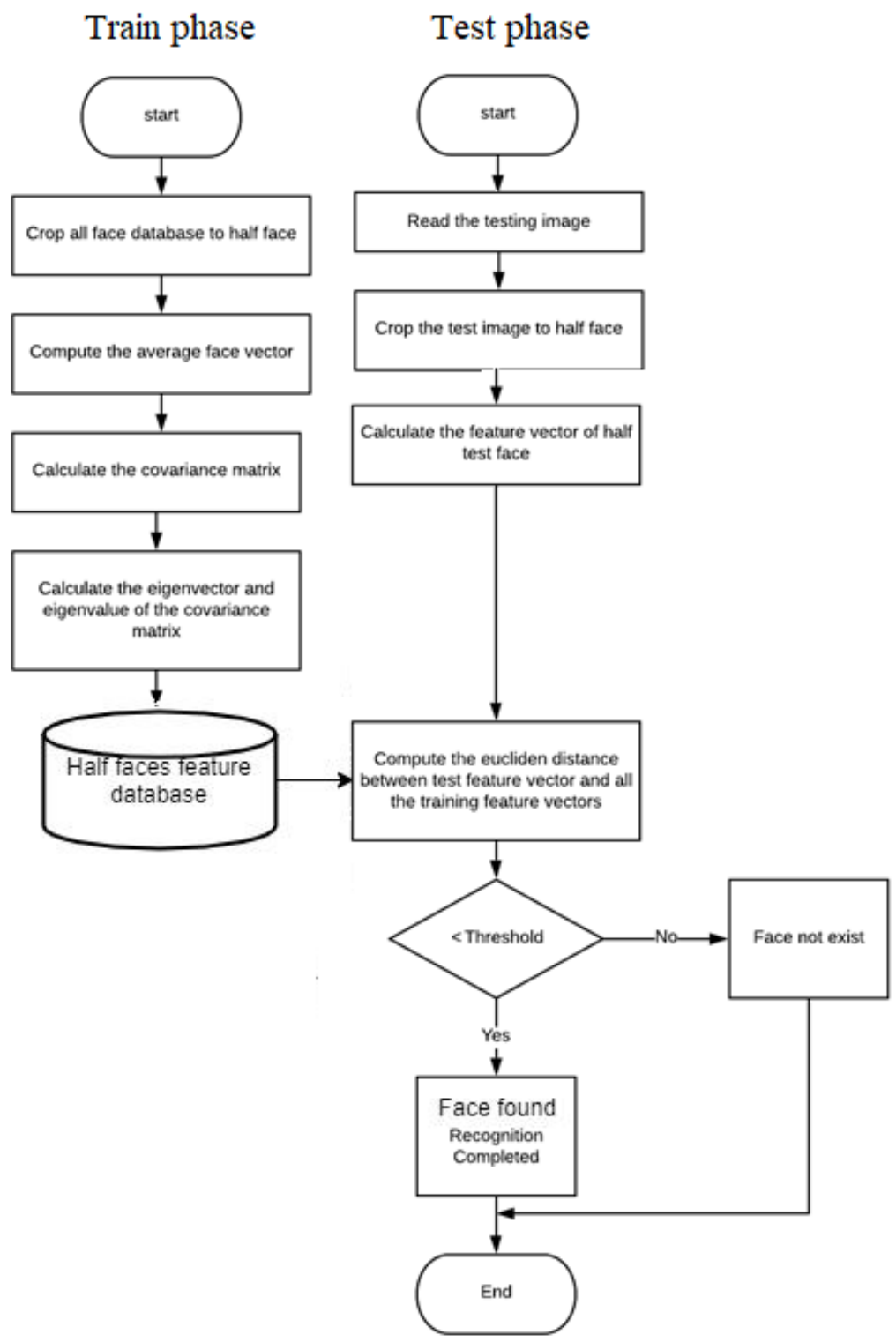

Figure 3. Proposed system flowchart

\subsection{Principal component analysis}

Principal component analysis (PCA) is a powerful method, it is used to extract the facial features using Eigenfaces. The purpose of the PCA method is primarily to simplify the variables observed and reduce their dimensions. The PCA also has two major advantages. First, the way of calculation of the covaraince matrix is simpler. Second, we don't need a lot of times. The following are the PCA step: 
- Construct the face image $\mathrm{N} \times \mathrm{N}$ of image I

- Read all face images

- Adjust the image dimensions to vector size $1 \times N^{2}$ and represent each image $I_{i}$ as vector $r_{i}$.

- Determine the mean matrix

$$
\psi=\frac{1}{m} \sum_{i=1}^{m} r_{i}
$$

- Substract from each vector matrix the mean matrix

$$
\phi=r_{i}-\psi
$$

- Determine the covariance matrix

$$
C=\phi \phi^{T}
$$

- Calculate eigenvalue and eigenvector

$$
C u_{i}=\lambda u_{i}
$$

Eigenvalue $(\lambda)=\operatorname{Det}\left(C-\lambda_{i}\right)$

Eigenvector $\left(u_{i}\right)=\left(C-\lambda_{i}\right) u_{i}$

- Calculate eigenface $(\mu)$

$$
\mu=\sum_{i=1}^{m} v_{i} Q_{i}
$$

Where $\psi$ is the mean matrix, $\phi$ the subtraction matrix, $C$ the covariance matrix, $\lambda$ Eigenvalue, $u_{i}$ Eigenvector and finally $\mu$ is the eigenface. As a result, large decrease in calculation is obtained, from the order of number of pixels in the images $N^{2}$ to the new order $M$, which is the number of images in the training set [22], [23].

\subsection{Euclidian distance}

We use the Euclidian Distance to measure the similarity between the input image and the data base images. It is widely used because of its simplicity. Euclidean Distance is described by the (7) as shown [24]:

$$
d(y, x)=\sqrt{\sum_{i=1}^{n}\left(x_{i}-y\right)^{2}}
$$

Where $n$ represent the features number, $\boldsymbol{y}$ represent the feature coefficient values of test image, $\boldsymbol{x}_{\boldsymbol{i}}$ is the feature coefficient values of database images and $d(y, x)$ is the Euclidean Distance between the test image vector and the database images vectors.

\section{IMPLEMENTATION AND TESTING}

The proposed approach implemented using MATLAB 2013a on Core i7-8550U CPU $2.00 \mathrm{GHz}$ and 8 GB of RAM running Windows 10 (64 bit) operating system. The proposed system is made up of three steps: pre-processing, features extraction and classification. PCA is used in extracting the feature of the image and Euclidian distance is used in classifying the extracted features. The first two principal component is show in Figure 4.

The experiment is applied in calculating the system performance on ORL database. This database is made up of 10 different images for 40 persons. Each image is gray with a resolution of $92 \times 112$ and a PGM format. Faces having different expressions and multiple poses are found in this database [25]. The data base was used for training. Other images that are not in database are enrolled is the testing phase to examine the effectiveness of the system in recognizing faces. Figure 5 shows the result for a person found in the data base and how is not.

The result showed that the accuracy of the system reached up to $96 \%$, the database is minimized by $46 \%$ and the computation time is decreased from 120 to $70 \mathrm{msec}$ with a $41.6 \%$ reduction. Due to this reduction the system can work more effectively in real time systems. In Tables 1 and 2 a comparition is presented with some previous work for the computation time and accuracy. It can be seen that the proposed system has good results both in computation time and accuracy as compared to others. 


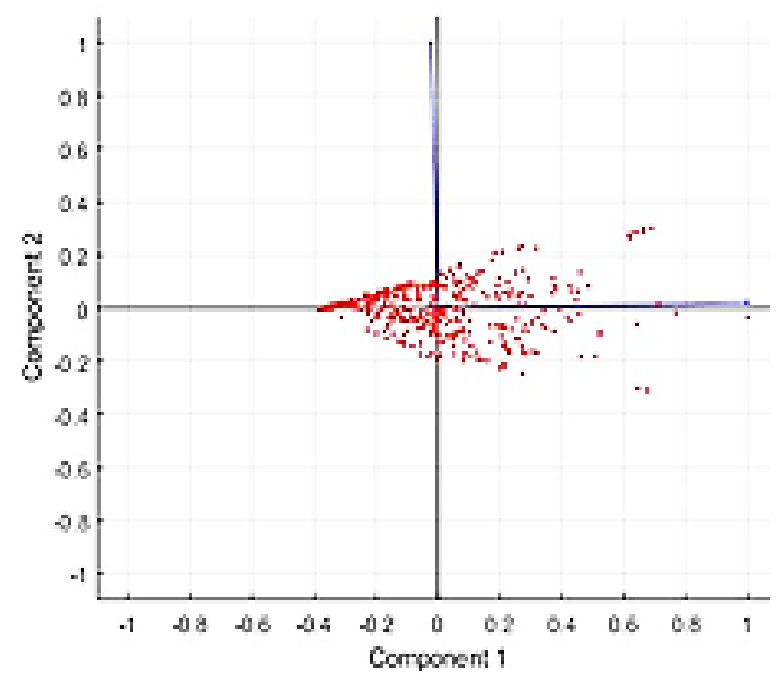

Figure 4. The first two principal component

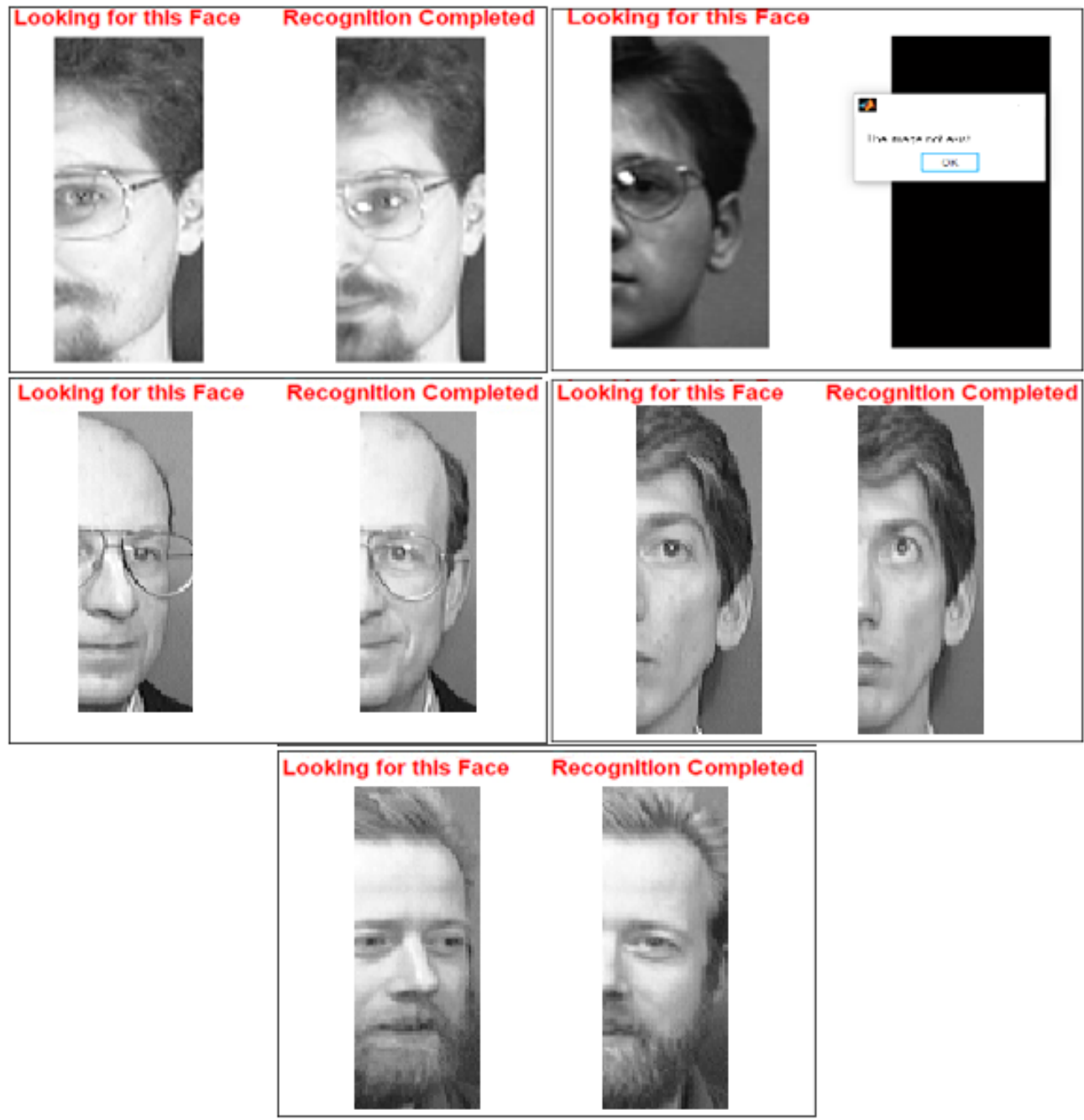

Figure 5. Examples of the system results 
Table 1. Comparison of proposed method computation time with existing methods

\begin{tabular}{ccc}
\hline Method & Full or half-face & Computation time $(\mathrm{msec})$ \\
\hline Sharma et al. $[19](2012)$ & Full & 384 \\
Sharma et al. $[19](2012)$ & Half & 99 \\
Proposed & Half & $\mathbf{7 0}$ \\
\hline
\end{tabular}

Table 2. Comparison of proposed method accuracy with existing methods

\begin{tabular}{lccc}
\hline Method & Full or Half-face & Recognitio Rate (\%) & Database \\
\hline Sharma et al. $[19]$ (2012) & Full & 83 & $3 \mathrm{D}$ \\
Sharma et al. [19] (2012) & Half & 95.3 & $3 \mathrm{D}$ \\
Shehzad et al. [20] (2014) & Full & 92 & Essex frontal \\
Shehzad et al. [20] (2014) & Half & 96.15 & Essex frontal \\
Halvi et al. $[8]$ (2017) & Full & 98.4 & ORL \\
Proposed & Half & $\mathbf{9 6}$ & ORL \\
\hline
\end{tabular}

\section{CONCLUSION}

In this paper, principal component analysis and eigenface approach is used to implement an accurate and robust system for face recognition in MATLAB environment. Starting from the motivation to reduce the database size and minimize the computation time, A number of half-face images are processed in the system and tested to recognize the person. The result proved that using half-face with the combination of principal component analysis and euclidian distance is effective in this face recognition system. The proposed system reduced the size of database and the speed of recognition with high degree of accuracy.

\section{REFERENCES}

[1] S. Zhang, Z. Li, and Y. Liu, "Research on face recognition technology based on PCA and SVM," Proceedings of the 11th International Conference on Modelling, Identification and Control (ICMIC2019), pp. 75-85, 2020, doi: 10.1007/978-981-15-0474-7 8.

[2] S. Sharma and K. Sachdeva, "Face Recognition using PCA and SVM with surf technique," International Journal of Computer Applications, vol. 129, no. 4, pp. 41-46, 2015.

[3] Z. Wang, et al., "Masked face recognition dataset and application," arXiv preprint arXiv, 2020.

[4] A. Choudhary and R. Vig, "Face recognition using multiresolution wavelet combining discrete cosine transform and Walsh transform," Proceedings of the 2017 International Conference on Biometrics Engineering and Application, pp. 33-38, 2017, doi: 10.1145/3077829.3077835.

[5] X. Fontaine, R. Achanta, and S. Süsstrunk, "Face recognition in real-world images," 2017 IEEE International Conference on Acoustics, Speech and Signal Processing (ICASSP), 2017, pp. 1482-1486, doi: 10.1109/ICASSP.2017.7952403.

[6] A. Singh, S. K. Singh, and S. Tiwari, "Comparison of face recognition algorithms on dummy faces," The International Journal of Multimedia \& Its Applications, vol.4, pp. 121-135, 2012, doi: 10.5121/ijma.2012.4411.

[7] Priyanka and Y. Singh, "A study on facial feature extraction and facial recognition approaches," International Journal of Computer Science and Mobile Computing, vol. 4, no. 5, pp. 166-174, 2014.

[8] S. Halvi, N. Ramapur, K. B. Raja, and S. Prasad, "Fusion based face recognition system using 1D transform domains," Procedia computer science, vol. 115, pp.383-390, 2017, doi: 10.1016/j.procs.2017.09.095.

[9] A. Alazzawi, O. N. Ucan, and O. Bayat, "Performance of face recognition system using gradient laplacian operators and new features extraction method based on linear regression slope," Mathematical Problems in Engineering, 2018, doi: 10.1155/2018/1929836.

[10] Y. Chu, L. Zhao, and T. Ahmad, "Multiple feature subspaces analysis for single sample per person face recognition," The Visual Computer, vol. 35, no. 2, pp. 239-256, 2019, doi: 10.1007/s00371-017-1468-4.

[11] R. S. Kute, V. Vyas, and A. Anuse, "Component-based face recognition under transfer learning for forensic applications," Information Sciences , vol. 476, pp. 176-191, 2019, doi: 10.1016/j.ins.2018.10.014.

[12] S. Hua, G. Chen, H. Wei, and Q. Jiang, "Similarity measure for image resizing using SIFT feature," EURASIP Journal on Image and Video Processing, 2012, doi: 10.1186/1687-5281-2012-6.

[13] E. Gumus, N. Kilic, A. Sertbas, and O. N. Ucan, "Evaluation of face recognition techniques using PCA, wavelets and SVM," Expert Systems with Applications, vol. 37, no. 9, pp. 6404-6408, 2010, doi: 10.1016/j.eswa.2010.02.079.

[14] S. Gutta, M. Trajkovic, and V. Philomin, "Face-recognition using half-face images," U.S. Patent US20030223623A1. Dec. 4, 2003.

[15] S. Gutta and H. Wechsler, "Partial faces for face recognition: Left vs right half," International Conference on Computer Analysis of Images and Patterns, vol 2756, pp. 630-637, 2003, doi: 10.1007/978-3-540-45179-2 77.

[16] Yi Zhu and F. Cutu, "Face Detection using Half-Face Templates," Journal of Vision, vol. 3, pp. 839-839, 2010, doi: 10.1167/3.9.839. 
[17] J. Harguess, S. Gupta and J. K. Aggarwal, "3D face recognition with the average-half-face," 2008 19th International Conference on Pattern Recognition, 2008, pp. 1-4, doi: 10.1109/ICPR.2008.4761503.

[18] W. Chen, T. Sun, X. Yang, and L. Wang, "Face detection based on half face-template," 2009 9th International Conference on Electronic Measurement \& Instruments, 2009, pp. 4-54-4-58, doi: 10.1109/ICEMI.2009.5274642.

[19] V. Sharma and R. Vashisht, "Average half face recognition by elastic bunch graph matching based on distance measurement," International Journal for Science and Emerging Technologies with Latest Trends, vol. 3, no. 1, pp. 24-35, 2012.

[20] M. Shehzad, M. Awais, M. Amin, and Y. A. Shah, "Face Recognition using average half face template," International Journal of Technology, vol. 5, no. 2, pp. 159-168, 2014, doi: 10.14716/ijtech.v5i2.408.

[21] University of Bradford, Half a face enough for recognition technology, ScienceDaily, May 2019. [Online]. Available: www.sciencedaily.com/releases/2019/05/190501114602.htm

[22] M. Turk and A. Pentland, "Eigen faces for recognition," Journal of CognitiveNeuroscience, vol. 3, no. 1, pp. 71-86, 1991, doi: 10.1162/jocn.1991.3.1.71.

[23] J. C. Sutherland and A. Parente, "Combustion modeling using principal component analysis," Proceedings of the Combustion Institute, vol. 32, no. 1, pp. 1563-1570, 2009, doi: 10.1016/j.proci.2008.06.147.

[24] Liwei Wang, Yan Zhang, and Jufu Feng, "On the Euclidean distance of images," in IEEE Transactions on Pattern Analysis and Machine Intelligence, vol. 27, no. 8, pp. 1334-1339, Aug. 2005, doi: 10.1109/TPAMI.2005.165.

[25] Face recognition homepage, Databases, 2005. [Online]. Availbale: https://www.face-rec.org/databases/

\section{BIOGRAPHIES OF AUTHORS}

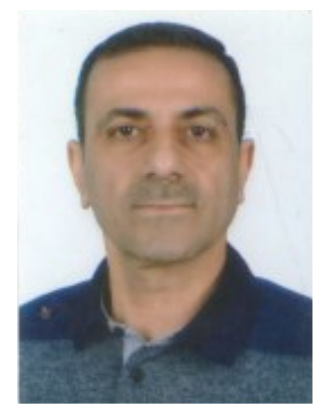

Ahmed M. Alkababji, received the B.Sc., M.Sc. and PhD degree in Electrical Engineering from University of Mosul, Iraq in 1994. 1996 and 2007. Currently, an assistant professor at computer engineering department in University of Mosul, Iraq. Research interest in signal processing, realtime systems and biometric engineering.

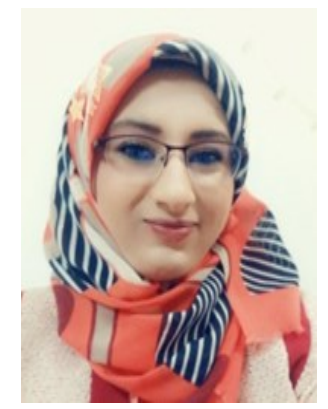

Sara Raed Abd, received the B.S. and M.S. degrees from the Department of Computer Engineering at the Mosul University in 2013 and 2018 respectively. She is currently working toward the $\mathrm{PhD}$ degree in Computer Engineering at the same university. She is interested in doing research in wireless sensor networks, cross-layer design for WSNs, Body Area Network, IoT, and biometric security. 